\title{
STRONG LIMITS RELATED TO THE OSCILLATION MODULUS OF THE EMPIRICAL PROCESS BASED ON THE K-SPACING PROCESS
}

\author{
GANE SAMB LO
}

\begin{abstract}
Aвstract. Recently, several strong limit theorems for the oscillation moduli of the empirical process have been given in the iid-case. We show that, with very slight differences, those strong results are also obtained for some representation of the reduced empirical process based on the (non-overlapping) $\mathrm{k}$-spacings generated by a sequence of independent random variables (rv's) uniformly distributed on $(0,1)$. This yields weak limits for the mentioned process. Our study includes the case where the step $\mathrm{k}$ is unbounded. The results are mainly derived from several properties concerning the increments of gamma functions with parameters $\mathrm{k}$ and one.
\end{abstract}

Nota-Bene. This paper was part of the $\mathrm{PhD}$ thesis at Cheikh Anta Diop University, 1991, not yet published in a peer-reviewed journal by August 2014. A slightly different version was published in Rapports Techniques, LSTA, Université Paris VI, 48, 1986, under the same title.

\section{IntroduCtion AND STATEMENT OF THE RESUlts}

Consider $U_{1}, \ldots, U_{n}$ a sequence of independent rv's uniformly distributed on $(0,1)$, and let

$$
U_{0, n}=0 \leq U_{1, n} \leq \ldots \leq U_{n, n} \leq U_{n+1, n}=1
$$

be their order statistics. The rv's

$$
D_{i, n}^{k}=U_{k i, n}-U_{(i-1) k, n}, 1 \leq i \leq\left[\frac{n+1}{k}\right]=N,
$$

where $[x]$ denotes the integer part of $x$, are called the non-overlapping $k$-spacings. Throughout, we shall assume that $N$ and $k$ are given and that $n$ is defined by $n=\inf \left\{j,\left[\frac{j+1}{k}\right]=N\right\}$ and then we will be able to study all our sequences as indexed by $N$ since $k$ will be either fixed or function of $N$.

Key words and phrases. Oscillation mudulus, empirical processes, increments of functions, law of the iterated logarithm, order statistics. 
The study of the properties of $D_{i, n}^{k}$ was introduced by Pyke [8] and several related papers have appeared in recent years (see e.g. [3]). One of the problem concerning the k-spacings is the study of the empirical process associated with $N k D_{i, n}^{k}, 1 \leq i \leq N$.

In order to give a comprehensible definition of that process, we recall the following representation which can be found in 11 in the case where $(n+1) / k$ is an integer :

$$
\left\{D_{i, n}^{k}, 1 \leq i \leq N\right\}={ }^{d}\left\{\frac{Y_{i}}{S_{n+1}}, 1 \leq i \leq N\right\}=:\left\{\frac{\left(\sum_{j=(i-1) k+1}^{j=i k} E_{i}\right)}{S_{n+1},}, 1 \leq i \leq N\right\},
$$

where $=^{d}$ denotes the equality in distribution and $S_{n}$ is the partial sum associated with $E_{1}, \ldots, E_{n}$, a sequence of independent and exponential rv's with meanone, i.e., $S_{n}=E_{1}+\ldots+E_{n}$. Thus, it follows that, if $\frac{(n+1)}{k}$ is an integer, the limiting distribution function of $\mathrm{Nk} D_{i, n}^{k}$, for any $\mathrm{i}$ and $\mathrm{k}$ fixed, is

$$
H_{k}(x)=\int_{0}^{x} \frac{t^{k-1} e^{-t}}{(k-1) !} d t, x \geq 0 .
$$

Therefore the empirical process (E.P.) associated with $N k D_{i, n}^{k}, 1 \leq i \leq$ $N$, may be defined by

$$
\beta_{N}(x)=N^{\frac{1}{2}}\left\{F_{N}(x)-H_{k}(x)\right\}, 0 \leq x \leq+\infty,
$$

where $F_{N}$ is the empirical distribution (E.D.F.) of $N k D_{i, n}^{k}, 1 \leq i \leq N$, with

$$
F_{N}(x)=\# \frac{\left\{i, 1 \leq i \leq N, N k D_{i, n}^{k} \leq x\right\}}{N}, x \geq 0 .
$$

Straightforward manipulations from (1.1), (1.2) and (1.3) as given in [1] show that even in the general case where $(N-1) k \leq n+1 \leq N k$, the reduced process $\alpha_{N}(s)=\beta_{N}\left(H_{k}^{-1}(s)\right), 0 \leq s \leq 1$, satisfies

$$
\left\{\alpha_{N}(s), 0 \leq s<1\right\}={ }^{d}\left\{N^{\frac{1}{2}}\left\{\xi_{N}\left(\delta_{n} H_{k}^{-1}(s)\right)-s\right\}+0\left(N^{-\frac{1}{2}}\right), 0 \leq s \leq 1\right\},
$$


where $H_{k}^{-1}$ is the inverse function of $H_{k}, \xi_{N}$ is the E.D.F. pertaining to $Y_{1}, \ldots, Y_{n}$ and $\delta_{n}=\frac{S_{n+1}}{N k}$.

The aim of this paper is to give the behavior of the oscillation modulus of $\alpha_{N}($.$) both where k$ is fixed and where $k \uparrow+\infty$. To this end we define

$$
\wedge_{N}\left(a_{N}, R_{N}\right)=\sup _{0 \leq h \leq a_{N}} \sup _{0 \leq s \leq 1-h}\left|R_{N}(s+h)-R_{N}(s)\right|
$$

and

$$
k_{N}\left(a_{N}, R_{N}\right)=\frac{\wedge_{N}\left(a_{N}, R_{N}\right)}{\left(2 a_{N} \log \log a_{N}^{-1}\right)^{\frac{1}{2}}},
$$

for any sequence of functions $R_{N}(s), 0 \leq s \leq 1$ and for any sequence $\left(a_{N}\right)_{N \geq 1}, 0<a_{N}<1$. The properties of $\wedge_{N}\left(a_{N}, R_{N}\right)$, the oscillation modulus of $R_{N}$, have been first described by Csörgo and Révèsz [2] and Stute [10] when $R_{N}$ represents the E.P. pertaining to a sequence of independent and uniformly distributed rv's with

$$
\begin{aligned}
& N a_{N} \rightarrow+\infty, \\
& \frac{\left(\log a_{N}^{-1}\right)}{\left(N a_{N}\right)} \rightarrow 0
\end{aligned}
$$

and

$$
\frac{\left(\log a_{N}^{-1}\right)}{\log \log N \rightarrow+\infty}
$$

as $N \rightarrow+\infty$.

Later, Mason, Shorack and Wellner (MSW) [7] dealt with the same for several choices of $\left(a_{N}\right)$ and give among the results an Erdös-Renyi law.

The chief achievement of this paper is the extension of those limit results to some sequence of process $\bar{\alpha}_{N}$ equal in distribution to $\alpha_{N}$. In fact, the fundemental role is played here by the properties of the tails of the gamma function $H_{k}^{\prime}($.$) , the derivative funtion of H_{k}$. These properties are established in Section 2 through technical lemmas and the proofs of the following results are given in Section 3. 
Theorem 1. Let $k$ be fixed. Then, there exists a sequence of processes $\alpha_{N}^{-}(s), 0 \leq s \leq 1, N=1,2, \ldots$ such that

$$
\forall N \geq 1,\left\{\alpha_{N}(s), 0 \leq s \leq 1\right\} d=\left\{\alpha_{N}^{-}(s), 0 \leq s \leq 1\right\} .
$$

(I) If $\left(a_{N}\right)_{N \geq 1}$ is a sequence of non-decreasing numbers satisfying the Csörgo-Révèsz-Stute conditions (S1), (S2) and (S3), then

$$
\lim _{N \uparrow+\infty} k_{N}\left(a_{N}, \alpha_{N}^{-}\right)=1 \text {, a.s. }
$$

(II) If

$$
a_{N}=c N^{-1} \log N, c>0, N \geq 1
$$

then

$$
\lim _{N \uparrow+\infty} k_{N}^{\circ}\left(a_{N}, \alpha_{N}^{-}\right)=\left(\frac{c}{2}\right)^{\frac{1}{2}}\left(\beta^{+}-1\right), \text { a.s., where } \beta^{+}>1
$$

and

$$
\beta^{+}\left(\log \beta^{+}-1\right)=c^{-1}-1
$$

$$
\operatorname{If} a_{N}=(\log N)^{-c}, c>0
$$

then

$c^{\frac{1}{2}} \leq \lim _{N \rightarrow+\infty} \inf k_{N}\left(a_{N}, \alpha_{N}^{-}\right) \leq \lim _{N \rightarrow+\infty} \sup k_{N}\left(a_{N}, \bar{\alpha}_{N}\right) \leq(1+c)^{\frac{1}{2}}$, a.s. (IV) If

$$
a_{N}=c_{N} N^{-1} \log N, c_{N} \rightarrow 0
$$

such that

$$
\left(c_{N} \log N\right)=N a_{N} \rightarrow+\infty
$$

and

$$
(\log N)^{-1}\left(\log c_{N}^{-1}\right) \log \log N \rightarrow 0 \text { as } N \uparrow+\infty
$$

then

$$
\lim _{N \uparrow+\infty} \sup \frac{N^{\frac{1}{2}} \log \left(\frac{1}{c_{N}}\right)}{\log N} \wedge_{N}\left(a_{N}, \bar{\alpha}_{N}\right) \leq 2 \text {, a.s. }
$$

We also have

Theorem 2. If $k=k(N) \rightarrow+\infty$ such that for some $\delta>2$ and for some $N_{0}$,

$$
0<a_{N} \leq t_{k}(\delta)=k^{k(\delta-2)} \exp \left(\frac{-k^{\delta}}{2}\right), N \geq N_{0}
$$

then Parts (I), (II), (III) and (IV) remain true. 
Remark 1. If each $\alpha_{N}$ is the spacings empirical process based on a sample depending on $N$, say $\chi(N)$, and if these samples $\chi(N)$, $N=1,2, \ldots$ are mutually independent (this statistical situation is quite concievable, for instance when checking homogeneity) the strong limit results of Theorem 1 are also valid for $\alpha_{N}$. One might seek other conditions to get the same extensions. Here, we restrict ourselves to weak extensions in the following 
Corollary 1. Let $k$ be either fixed or $k \rightarrow+\infty$. Let $\left(a_{N}\right)_{N \geq 1}$ be a sequence of positive numbers such that $0 \leq a_{N} \leq t_{k}(\delta)$ when $N \geq N_{0}$, for some $N_{0}$ and $\delta>2$. Then:

(I) Under the assumptions of Part I of Theorem 1, we have

$$
\lim _{N \rightarrow+\infty} k_{N}\left(a_{N}, \alpha_{N}\right)=1 \text { in probability. }
$$

(II) Under the assumptions of Part II of Theorem 1, we have

$$
\lim _{N \rightarrow+\infty} k_{N}\left(a_{N}, \alpha_{N}\right)=\left(\frac{c}{2}\right)^{\frac{1}{2}}\left(\beta^{+}-1\right) \text { in probability }
$$

(III) Under the assumptions of Part III of Theorem 1, we have

$$
\lim _{N \rightarrow+\infty} k_{N}\left(a_{N}, \alpha_{N}\right)=c^{\frac{1}{2}} \text {, in probability. }
$$

(IV) Under the assumptions of Part IV of Theorem 1, we have

$$
\lim _{N \rightarrow+\infty} P\left(\frac{N^{\frac{1}{2}} \log \left(\frac{1}{c_{N}}\right)}{\log N}\right) \wedge_{N}\left(a_{N}, \alpha_{N}\right)>(2+\varepsilon)=0 \text { for all } \varepsilon>0 .
$$

Remark 2. It appears from Theorems 1 and 2 that the oscillation modulus of $\bar{\alpha}_{N}$ and that of the uniform empirical process are almost the same. In [5], we prove that the exact strong bounds in (I) and (II) reamin for $\alpha_{N}$ when $a_{N}$ satisfies further conditions.

Remark 3. One might think that deriving the result of our corollary by using invariance principles (as given in [1] and [5]) and well-known results for the Brownian bridge would be easier (at least for some sequences $a_{N}$ ). This is not true at all (see Remark 4 below).

\section{TECHNiCAL LEMMAS}

It will follow from Lemma 1 of section 3 that the increments of $\bar{\alpha}_{N}$ behave as the increments of $\gamma_{N}(\psi()$.$) and those of \phi($.$) where \gamma_{N}($.$) is$ the E.P. pertaining to

$$
\begin{gathered}
U_{1}, \ldots, U_{N}, \psi(s)=H_{k}\left(\mu_{n} H_{k}^{-1}(s)\right), 0 \leq s \leq 1, \mu_{n} d_{=} \delta_{n}, \\
n=1,2, \ldots, \phi(s)=H_{k}^{\prime}\left(H_{k}^{-1}(s)\right) H_{k}^{-1}(s), 0 \leq s \leq 1,
\end{gathered}
$$

with $H_{k}^{\prime}(x)=\frac{d H_{k}(x)}{d x}$, for all positive $\mathrm{x}$. Then, since $k_{N}(., \gamma N)$ is known, our study is reduced to describing the increments of $\psi($.$) and that of$ $\phi($.$) , what we do in this paragraph.$ 
Lemma 1. Let $k$ be fixed and $a=a_{N}$ be a sequence of positive numbers satisfying

$$
\left(n^{-1} \log \log n\right)^{\frac{1}{2}} \log \left(\frac{1}{a}\right) \rightarrow 0 \text { as } N \rightarrow+\infty \text { and } a \rightarrow 0,
$$

then as $N \rightarrow++\infty$, we have the following properties

$$
\sup _{0 \leq h \leq a} \sup _{0 \leq s \leq 1-h}|\psi(s+h)-\psi(s)|=a(1+o(1)) a . s .,
$$

uniformly in $s$,

$$
0 \leq s \leq 1-a,|\psi(s+a)-\psi(s)|=a(1+q(a)),
$$

where $q(a) \rightarrow 0$, a.s., as $a \rightarrow 0$.

\section{Proof of lemma 1 .}

We need several properties of gamma functions. First note that for a fixed $\mathrm{k}$,

$$
s=1-H_{k}(s)=\frac{e^{-x} x^{k-1}}{(k-1) !}\left\{1+\frac{k-1}{x}+\frac{k-2}{x^{2}}+\ldots+\frac{(k-a) !}{x^{k-1}}\right\},
$$

and

$$
\begin{gathered}
x=H_{k}^{-1}(1-s)=\log \left(\frac{1}{s}\right)-\log (k-1) !+(k-1) \log x \\
+\log \left(1+\frac{k-1}{x}+\ldots+\frac{(k-1) !}{x^{k-1}}\right)
\end{gathered}
$$

from $k-1$ integrations by parts. Next for a fixed $\mathrm{k}$ or for $k \rightarrow+\infty$, we have, as $x \downarrow 0$,

$$
s=H_{k}(x)=\frac{x^{k}}{k !}(1+0(x))
$$

and

$$
x=H_{k}^{-1}(s)=(k !)^{\frac{1}{k}}\left(1+O\left(\frac{x}{k}\right)\right),
$$

where for any function $g(),. g(x)=O(y)$ as $x \downarrow 0$ means that $\lim _{x \downarrow 0} \sup \left|\frac{g(x)}{y}\right|<$ $+\infty$. To see this, use the following inequalities:

$$
0 \leq t \leq x \Rightarrow e^{-x} \leq e^{-t} \leq 1
$$


to obtain that $e^{-x} \frac{x^{k}}{k !} \leq H_{k}(x) \leq \frac{x^{k}}{k !}$ and the results follow. Now, we are able to prove lemma A1.

Let us continue the proofs Lemma 1. Define

$$
\Psi_{h}(s)=\psi(s+h)-\psi(s), 0 \leq s \leq 1-h, 0 \leq h \leq a, h=1,2, \ldots
$$

Straighforward computations give

$$
\frac{d \Psi_{h}(s)}{d s}=\mu_{n}^{k-1}\left\{\exp \left(\left(\mu_{n}-1\right) H_{k}^{-1}(s+h)\right)-\exp \left(\left(\mu_{n}-1\right) H_{k}^{-1}(s)\right)\right\} \text {. }
$$

Thus, for each elementary event $\omega$ of the probability space, for each $\mathrm{N}$ (that is to say for each $\mathrm{n}$ ) and for each $\mathrm{h}, \Psi_{h}$. (.) is non-decreasing of non-increasing according to the sign of $\mu_{n}(\omega)-1$. Thus we have

$$
\sup _{0 \leq s \leq 1-h}\left|\Psi_{h}(s)\right|=\max \left\{\Psi_{h}(0),\left|\Psi_{h}(1-h)\right|\right\} .
$$

Computation of $\Psi_{h}(1-h)$. By using (2.3) and (2.4), with $h=1-$ $H_{k}(x)$, we have

$$
\begin{aligned}
\mu_{n} H_{k}^{-1}(1-h)= & \mu_{n} \log \left(\frac{1}{h}\right)-\mu_{n} \log (k-1) !+\mu_{n}(k-1) \log x \\
& +\mu_{n} \log \left(1+\ldots+\frac{(k-1) !}{x^{k-1}}\right)
\end{aligned}
$$

Now recall that $\Psi_{h}(1-h)=1-H_{k}\left(\mu_{n} H_{k}^{-1}(1-h)\right)$ and, using (2.3), get

$$
\Psi_{h}(1-h)=\mu_{n}^{k-1} \frac{\left(\log \left(\frac{1}{h}\right)\right)^{k-1} h^{\mu_{n}}((k-1) !)^{\mu_{n}}}{(k-1) !} x^{-(k-1) \mu_{n}}\left(1+q_{1}(h)\right),
$$

where there exists $A_{k}$ and $B_{k}$ depending only on k (k being fixed) such that

$$
\left|q_{1}(h)\right| \leq A_{k} x^{-1} \log x+B_{k} x^{-1} \text {, as } h \rightarrow 0 \text { (i.e. as } x \rightarrow+\infty \text { ). }
$$

These constants $A_{k}$ and $B_{k}$ are provided by the approximation 


$$
\left|\frac{\log \left(\frac{1}{h}\right)}{x}-1\right| \leq A_{k} x^{-1} \log x+B_{k} x^{-1},
$$

as $h \rightarrow 0, x=H_{k}^{-1}(1-h) \rightarrow+\infty$. And (2.11) leads to

$$
\Psi_{h}(1-h)=h^{M U_{n}}\left(\log \left(\frac{1}{h}\right)\right)^{(k-1)\left(1-\mu_{n}\right)}\left(1+q_{2}(h)\right)^{-(k-1) \mu_{n}+1}\left(1+q_{3}(N)\right),
$$

where $q_{2}($.$) satisfies( 2.11) with the same constants \mathrm{A}_{k}$ and $\mathrm{B}_{k}$ and $q_{3}(N)=o(1)$, a.s., independently of $h, 0 \leq h \leq 1$, as $N \rightarrow+\infty$. Since the functions $x^{-1} \log x$ and $x^{-1}$ are non-increasing as $x \rightarrow+\infty$, it follows from (2.10), (2.11) and (2.12) that

$$
\forall 0 \leq h \leq a, \Psi_{h}(1-h)=(1+q(a)) h^{\mu_{n}}\left(\log \left(\frac{1}{h}\right)\right)^{(k-1) \mu_{n}},
$$

where $\mathrm{q}(a) \rightarrow 0$ and $\mathrm{N} \rightarrow+\infty$. By convention, we shall write $g(h)=$ $q(a)$ for $0 \leq h \leq a$, for all $h, 0 \leq h \leq a, g(h)=o(1)$ where the "o (1)" depends only on $a$, as a $\rightarrow 0$.

Computation of $\Psi_{n}(0)$.

We have $\Psi_{h}(0)=H_{k}\left(\mu_{n} H_{k}^{-1}\right)$. Then by using (2.5) -(2.6), we obtain

$$
\mu_{n} H_{k}^{-1}(h)=\mu_{n}(k !)^{\frac{1}{k}} h^{\frac{1}{k}}(1+q(a)), 0 \leq h \leq a .
$$

Use again (2.5)-(2.6) and get $H_{k}\left(\mu_{n} H_{k}^{-1}(h)\right)=h \mu_{n}^{k}(1+q(a))$, a.s., $0 \leq h \leq a \rightarrow 0$, since $\mathrm{k}$ is fixed and $\mu_{n} \rightarrow 1$, a.s., as $N \rightarrow+\infty$. Then,

$$
\sup _{0 \leq h \leq a} \sup _{0 \leq s \leq 1-h} \Psi_{h}(s)=(1+q(a)) \sup _{0 \leq h \leq a} \max \left\{h, h^{\mu_{n}}\left(\log h^{-1}\right)^{(k-1)\left(1-\mu_{n}\right)}\right\},
$$

a.s., and $N \rightarrow+\infty$. But,

$$
\begin{gathered}
\forall N \geq 1, \frac{d\left\{h^{\mu_{n}}\left(\log h^{-1}\right)^{(k-1)\left(1-\mu_{n}\right)}\right\}}{d h}=h^{\mu_{n}}\left(\log h^{-1}\right)^{(k-1)\left(1-\mu_{n}\right)} \\
\quad \times\left\{\mu_{n}-\frac{(k-1)\left(1-\mu_{n}\right)}{\log h^{-1}}\right\}
\end{gathered}
$$


Thus $h^{\mu_{n}}\left(\log h^{-1}\right)^{(k-1)\left(\mu_{n}-1\right)}$ is non-decreasing when $n$ sufficiently large since $k\left(1-\mu_{n}\right) \rightarrow 0$, a.s., as $N \rightarrow+\infty$ by the strong law of large numbers ( $k$ being fixed).Then,

$$
0 \leq h \leq a \Rightarrow h^{\mu_{n}}\left(\log h^{-1}\right)^{(k-1)\left(1-\mu_{n}\right)} \leq a^{\mu_{n}}\left(\log a^{-1}\right)^{(k-1)\left(1-\mu_{n}\right)},
$$

a.s., as $N \rightarrow+\infty$. Furthermore,

$$
\left(\log a^{-1}\right)^{(k-1)\left(1-\mu_{n}\right)}=\exp \left((k-1)\left(1-\mu_{n}\right) \log \log a^{-1}\right)=1+o(1),
$$

a.s., whenever $\left(1-\mu_{n}\right) \log \log a^{-1} \rightarrow 0$, a.s. But this is implied by (Q1). Indeed, we have by the law of the iterated logarithm (the loglog law) that

$$
\lim _{N \rightarrow+\infty} \sup \left(2 n^{-1} \log \log n\right)^{\frac{1}{2}}\left|\mu_{n}-1\right| \leq 1, \text { a.s. }
$$

This together with (Q1) imply that $\left(1-\mu_{n}\right) \log \log a^{-1} \rightarrow 0$, a.s., as $N \rightarrow+\infty$. In fact, the loglog law holds for $\delta_{n}$, that is

$$
\lim _{N \rightarrow+\infty} \sup \left(2 n^{-1} \log \log n\right)^{\frac{1}{2}}\left|\delta_{n}-1\right| \leq 1 \text {, a.s. }
$$

But (2.21) may be obtained from (see [5], Appendix)

$$
\sum_{p \geq 0} P\left(\bigcup_{n_{p}}^{n_{p+1}-1}\left\{\left(2 n^{-1} \log \log n\right)\left|\delta_{n}-1\right| \geq 1+\frac{\varepsilon}{2}\right\}\right)<+\infty
$$

where $\left(n_{p}\right)$ is an increasing and unbounded sequence of positive integers and $\varepsilon>0$ is arbitrary. This and the equality in distribution of $\delta_{n}$ and $\mu_{n}$ for each $\mathrm{N}$ imply (2.20). The same loglog - law shows that (Q1) implies that

$$
a^{1-\mu_{n}}=\exp \left(\left(1-\mu_{n}\right) \log a^{-1}\right)=1+o(1) a s N \rightarrow+\infty .
$$

We finally get from (2.13), (2.18), (2.19) and (2.22) that

$$
\sup _{0 \leq h \leq a} \sup _{0 \leq s \leq 1-h}\left|\Psi_{h}(s)\right|=a(1+o(1)) \text { a.s., } a d N \rightarrow+\infty,
$$

which proves Part (2.1) of Lemma 1. To prove part (2.2), it suffices to remark that we may have through (2.8) that 
$\min \left(\phi_{a}(0), \phi_{a}(1-a)\right) \leq \phi_{a}(s) \leq \max \left(\phi_{a}(0), \phi_{a}(1-a)\right), 0 \leq s \leq-a$, and the part in question follows since the first part implies that $\phi_{a}(0)=$ $a(1+o(1))$, a.s. and $\phi_{a}(1-a)=a(1+o(1))$, a.s., as $N \rightarrow+\infty$.

Lemma 2. Let $k$ be fixed, then we have as $a \rightarrow 0, N \rightarrow+\infty$,

$$
\sup _{0 \leq h \leq a} \sup _{0 \leq s \leq 1-h}|\phi(s)-\phi(s+h)|=\left(a \log a^{-1}\right)(1+o(1)) .
$$

\section{Proof of Lemma 2 .}

Consider $\Phi_{h}(s)=\phi(s+h)-\phi(s), 0 \leq s \leq 1-h$. Direct considerations yield that

$$
\frac{d \Phi_{h}(s)}{d s}=H_{k}^{-1}(s)-H_{k}^{-1}(s+h), 0 \leq s \leq 1-h .
$$

Then for each $\mathrm{h}, \Phi_{h}($.$) is non-increasing and thus,$

$$
\sup _{0 \leq s \leq 1-h}\left|\Phi_{h}(s)\right|=\max \left\{\left|\Phi_{h}(0)\right|,\left|\Phi_{h}(1-h)\right|\right\} .
$$

But, by (2.5)-(2.6),

$$
\Phi_{h}(0)=H_{k}^{\prime}\left(H_{k}^{-1}(h)\right) H_{k}^{-1}(h)=k h(1+q(a)),
$$

$0 \leq h \leq a \rightarrow 0$. Here we omit the details concerning the uniform approximations which provide $q($.$) . These details are very similar to$ those of the computation of $\Phi_{h}(0)$. By the considerations that were previously used for getting (2.10) from (1.1), we have

$\Phi_{h}(1-h)=H_{k}^{\prime}\left(H_{k}^{-1}(1-h)\right) H_{k}^{-1}(1-h)=h \log h^{-1}(1+q(a)), 0 \leq h \leq a \rightarrow 0$.

Notice that $H_{k}^{\prime}\left(H_{k}^{-1}(1-h)\right)$ yields something like (2.10) while $H_{k}^{-1}(1-h)$ yields $\left(\log h^{-1}\right)(1+q(a)), 0 \leq h \leq a \rightarrow 0$. We obtain

$\sup _{0 \leq h \leq a} \sup _{0 \leq s \leq 1-h}\left|\Phi_{h}(s)\right|=1+q(a) \sup _{0 \leq h \leq a} \max \left(k h, h \log h^{-1}\right)=\left(1+q(a)\left(a \log a^{-1}\right)\right)$, $a \rightarrow 0$, since $k$ is fixed here. Hence Lemma 2 is proved.

Now, we concentrate on the case where $k \rightarrow+\infty$, First, we give the following 
Proposition 1. Let

$$
0 \leq s \leq t_{k}(\delta)=k^{k(\delta-2)} \exp \left(-\frac{1}{2} k^{\delta}\right),
$$

Then, as $k \rightarrow+\infty$, we have

$$
x=H_{k}^{-1}(1-s)=\left(\log s^{-1}\right)\left(1+q_{4}(s)\right),
$$

where there exist $A$ and $k_{0}$ such that $\left|q_{4}(s)\right| \leq A \frac{\log k}{k^{\delta-1}}$ for all $0 \leq s \leq$ $t_{k}(\delta), k \geq k_{0}$.

Proof. Integrating by parts, we get

$$
\forall x \geq 0, \frac{x^{k-1} e^{-x}}{(k-1) !} \leq 1-H_{k}(x) \leq \frac{x^{k-1} e^{-x}}{(k-1) !}+\frac{k}{x}\left(1-H_{k}(x)\right)
$$

Then,

$$
\frac{x^{k-1} e^{-x}}{(k-1) !} \leq 1-H_{k}(x) \leq\left\{1-\frac{k}{x}\right\}^{-1} \frac{x^{k-1} e^{-x}}{(k-1) !}, \forall x \geq 0 .
$$

We are able to see that the expansion of $H_{k}(x)$ is then possible if $k / x \rightarrow 0$. Now, let $0 \leq s \leq s_{k}=1-H_{k}\left(k^{\delta}\right)$. Apply (2.25) and get

$$
0 \leq s \leq s_{k} \Rightarrow s=1-H_{k}(x)=\frac{x^{k-1} e^{-x}}{(k-1) !}\left\{1+q_{5}(x)\right\},
$$

with $\left|q_{5}(x)\right| \leq\left(1-k^{-(\delta-1)}\right) k^{-(\delta-1)}$ for all $0 \leq s \leq s_{k}$. But

$$
s_{k}=\frac{k^{\delta(k-1)} e^{-k}}{(k-1) !}\left(1+0\left(k^{-(\delta-1)}\right)\right) .
$$

Then by Sterling's formula and some sthraighforward calculations, it is possible to find a $k_{1}$ such that $t_{k}(\delta)=k^{k(\delta-2)} \exp \left(-\frac{1}{2} k^{\delta}\right) \leq s_{k}$ for all $k \leq k_{1}$. Then for $0 \leq s \leq s_{k}, k \geq k_{1}$,

$$
x=H_{k}^{-1}(1-s)=\left(\log s^{-1}\right)-\log (k-1) !+(k-1) \log x+o\left(q_{5}(s)\right) .
$$

Now since

$$
\begin{aligned}
& 0 \leq s \leq s_{k} \Rightarrow\left|\frac{(k-1) \log x}{x}\right| \leq \frac{\log k}{k^{\delta-1}}=0\left(k^{-(\delta-1)} \log k\right), \\
& 0 \leq s \leq s_{k} \Rightarrow\left|\frac{\log (k-1) !}{x}\right| \leq \frac{\log k !}{k^{\delta}}=0\left(k^{-(\delta-1)} \log k\right),
\end{aligned}
$$

by Sterling's formula. Thus, these two facts and (2.26) together imply that

$\forall s \leq s_{k}, \log s^{-1}=x\left(1+0\left(k^{-(\delta-1)} \log k\right)=H_{k}^{-1}(1-s)\left(1+0\left(k^{-\delta+1} \log k\right)\right)\right.$, 
which was to be proved. We finally give two lemmas which correspond to Lemmas 1 and 2 in the case of infinite steps $k$.

Lemma 3. Let $k$ satisfy, as $N \rightarrow+\infty$,

$$
(K) k N^{-1}(\log \log n) \rightarrow 0
$$

and

$$
k=k(N) \rightarrow+\infty
$$

Then the following assertions hold.

$$
\sup _{0 \leq h \leq a} \sup _{0 \leq s \leq 1-h}|\psi(s+h)-\psi(s)|=a(1+o(1)) \text {, a.s., as } N \rightarrow+\infty \text {. }
$$

$|\psi(s+h)-\psi(s)|=a(1+q(a))$, for $0 \leq s \leq 1-a$, with $q(a) \rightarrow 0$, a.s., as $N \rightarrow+\infty$.

Proof of of Lemma 3. As in Lemma 1, we have

$$
\sup _{0 \leq s \leq 1-h}\left|\psi_{h}(s)\right|=\max \left\{\left|\psi_{h}(0)\right|,\left|\psi_{h}(1-h)\right|\right\} \text {. }
$$

First we treat $\psi_{h}(0)=H_{k}\left(\mu_{n} H_{k}^{-1}(h)\right)$. Equations (2.5) $-(2.6)$ yield

$$
\mu_{n} H_{k}^{-1}(h)=(k !)^{\frac{1}{k}}\left(1+o\left(H_{k}^{-1} \frac{(a)}{k}\right)\right) \mu_{n}, 0 \leq h \leq a .
$$

Now we note that $0 \leq s \leq a$ implies that $0 \leq H_{k}^{-1}(h) \leq C_{1} a^{\frac{1}{k}}(k !)^{\frac{1}{k}}$ for small values of a, $C_{1}$ being a constant. Sterling's formula then implies for large values of $\mathrm{k}$,

$$
0 \leq s \leq t_{k}, 0 \leq H_{k}^{-1}(h) \leq \text { Const. } k^{\delta-1} \exp \left(-\frac{1}{2} k^{\delta-1}\right) .
$$

Then $H_{k}^{-1}(h) \rightarrow 0$ and we are able to use (2.5) -2.6 to get

$$
\forall 0 \leq h \leq a \Rightarrow H_{k}\left(\mu_{n} H_{k}^{-1}(h)\right)=\mu_{n}^{k} h\left(1+o\left(H_{k}^{-1}(a)\right) .\right.
$$

The loglog - law implies that

$$
k\left(1-\mu_{n}\right)=0\left(k\left(2 n^{-1} \log \log n\right)^{2}\right), \text { a.s. }
$$

Thus, whenever $(K)$ is satisfied, one has

$$
\mu_{n}^{k}=\exp \left(-k\left(1-\mu_{n}\right)(1+o(1)) \rightarrow 1\right. \text {, a.s. }
$$


Hence

$(2.30)$

$\forall 0 \leq s \leq a \leq t_{k}(\delta), \psi_{h}(0)=h(1+q(a)) \leq a(1+q(a))=\psi_{a}(0)$.

We now treat $\psi_{h}(1-h)$. By the proposition, we get

$\forall 0 \leq h \leq a \leq t_{k}(\delta), X=\mu_{n} H_{k}^{-1}(1-h)=\mu_{n}\left(\log h^{-1}\right)\left(1+0\left(\frac{\log k}{k^{\delta-1}}\right)\right)$,a.s.

Since $\frac{X}{k}=0\left(k^{-(\delta-1)}\right)$,a.s., one has

$1-H_{k}(X)=\mu_{n}^{k-1} \frac{\left(\log h^{-1}\right)^{k-1}}{(k-1) !}\left(1+0\left(\frac{\log k}{k^{\delta-2}}\right)\right) h^{\mu_{n}} x^{-(k-1) \mu_{n}}((k-1) !)^{\mu_{n}}\left(1+q_{4}(h)\right)$, a.s.,

as $N \rightarrow+\infty$. Replace $\mathrm{x}$ by $\log h^{-1}$ in (2.32). On account of (2.31) and

of the fact that $\left(1+0\left(k^{1-\delta} \log k\right)^{k-1}\right)=\left(1+0\left(k^{2-\delta} \log k\right)\right)$, we get

$$
\psi_{h}(1-h)=((k-1) !)^{1-\mu_{n}}\left(\log h^{-1}\right)^{(k-1)\left(1-\mu_{n}\right)} h^{\mu_{n}}(1+q(a)) .
$$

Finally, by taking $(K)$ and $(Q 2)$ into account, we find ourselves in the same situation as in the proof of Lemma 1 (see Statement (2.12)). But in order to have the same conclusion, i.e.,

$$
\sup _{0 \leq h \leq a} \psi_{h}(1-h)=a(1+q(a)), \text { a.s., as } N \rightarrow+\infty,
$$

we have to check that

$((k-1) !)^{1-\mu_{n}}=\exp \left(\left(1-\mu_{n}\right) \log (k-1) !\right)=: \rho_{n} \rightarrow 1$, a.s., as $N \rightarrow+\infty$.

But the loglog - law and Sterling's formula together show that

$$
\rho_{n}=\exp \left(0\left(\left(\frac{k^{\frac{1}{4}}(\log \log n)^{\frac{1}{2}}}{N^{\frac{1}{2}}} \log a^{-1}\right)\left(\frac{\log k}{\log a^{-1}}\right)\right)\right) .
$$

Obviously the condition $0<a \leq t_{k}(\delta)$ implies that $(\log k) /\left(\log a^{-1}\right) \rightarrow$ 0 as $N \rightarrow+\infty$, and as $k \rightarrow+\infty$. This fact combined with (Q2) clearly shows that $\rho_{n} \rightarrow 1$ as $N \rightarrow+\infty$. Now, by putting together (2.29), (2.30) and (2.33), we get

$\left(0<a \leq t_{k}(\delta), \delta>2\right) \Rightarrow \sup _{0 \leq 0 \leq a} \sup _{0 \leq s \leq 1-h}\left|\psi_{h}(s)\right|=a(1+q(a))$,a.s., as $N \rightarrow+\infty$.

Lemma 4. Let $0<a \leq t_{k}(\delta), \delta>2$. Then as $k \rightarrow+\infty$, we have $\sup _{0 \leq h \leq a} \sup _{0 \leq s \leq 1-h}|\phi(s+h)-\phi(s)|=\left(a \log a^{-1}\right)(1+q(a)), q(a) \rightarrow 0$ as $N \rightarrow+\infty, a \rightarrow 0$. 
Proof of of Lemma 4. If we proceed as in Lemma A2 and as in Lemma A3, we get

$\sup _{0 \leq h \leq a} \sup _{0 \leq s \leq 1-h}\left|\Phi_{h}(s)\right|=\max \left(k a,\left(a \log a^{-1}\right)\right)(1+q(a))$, as $N \rightarrow+\infty, a \rightarrow 0$

From there, the conclusion is obtained by noticing that the condition $0<a \leq t_{k}(\delta)$ implies that $\frac{\left(\log a^{-1}\right)}{k} \rightarrow+\infty$ as $k \rightarrow+\infty$.

\section{Proofs of the Results}

Throughout, we shall use the following representation which follows from [5] (see e.g. the study of $R_{N 1}(x)$ ).

Lemma 5. Let $k$ be fixed of $k \rightarrow+\infty$ as $N \rightarrow+\infty$, then

$$
\begin{aligned}
\left\{\alpha_{N}(s), 0 \leq s \leq 1\right\} & d=\left\{\gamma_{N}(\psi(s))+N^{\frac{1}{2}}\left\{H_{k}\left(\mu_{n} H_{k}^{-1}(s)\right)-s\right\}, 0 \leq s \leq 1\right\} \\
& =:\left\{\bar{\alpha}_{N}(s), 0 \leq s \leq 1, \text { a.s. }\right\}
\end{aligned}
$$

Lemma 5 will be systematically used. Then, if $a_{N}$ satisfies

$$
\lim _{N \rightarrow+\infty} \frac{(\log \log n)^{2}}{N a_{N} \log a_{N}^{-1}}=0,
$$

we will be able to focus our attention on $\gamma_{N}(\psi(s))+N^{\frac{1}{2}}\left(\mu_{n}-1\right) \phi(s)$ in the following way

$$
\begin{gathered}
\frac{\bar{\alpha}_{N}(s)}{b_{N}}=\frac{\gamma_{N}(\psi(s))}{b_{N}}+\frac{N^{\frac{1}{2}}\left(\mu_{n}-1\right) \phi(s)}{b_{N}}+b_{N}^{-1} 0\left(N^{\frac{1}{2}} \log \log n\right), \text { a.s. } \\
=: A_{N 1}(s)+A_{N 2}(s)+A_{N 3}(s) .
\end{gathered}
$$

with $b_{N}=\left(2 a_{N} \log \log a_{N}^{-1}\right)^{\frac{1}{2}}=b\left(a_{N}\right)$. It follows that if $(Q 3)$ holds we have $A_{N 3}(s)=o(1)$, a.s., uniformly with respect to $s, 0 \leq s \leq 1$.

Proof of Part I of Theorem 1, By (2.34), we have

$$
k_{N}\left(a_{N}, \bar{\alpha}_{N}\right) \leq k_{N}\left(a_{N}, A_{N 1}\right)+k_{N}\left(a_{N}, A_{N 2}\right)+k_{N}\left(a_{N}, A_{N 3}\right),
$$
and by Lemma 3, we have for a fixed $k$,

$$
k_{N}\left(a_{N}, A_{N 2}\right) \leq N^{\frac{1}{2}}\left|1-\mu_{n}\right| b\left(a_{N}\right)(1+o(1)) \text {, a.s. }
$$

as $N \uparrow+\infty$. Thus the loglog - law implies that

$$
\lim _{N \rightarrow+\infty} k_{N}\left(a_{N}, A_{N 2}\right)=o(1), \text { a.s. }
$$


whenever

$$
k^{-\frac{1}{2}}(2 \log \log n)^{\frac{1}{2}} b\left(a_{N}\right) \rightarrow 0 \text { as } N \rightarrow+\infty
$$

is satisfied. On the other hand, Lemma 1 and Theorem 0.2 of Stute [10] together yield that

$k_{N}\left(a_{N}, A_{N 1}\right) \leq k_{N}\left(a_{N}, \gamma_{N}\right)(1+o(1))=1+o(1)$,a.s., as $N \rightarrow+\infty$

Then if $(Q 1),(Q 3),(Q 4),(S 1)$ and $(S 3)$ are satisfied, we get

$$
\lim _{N \rightarrow+\infty} k_{N}\left(a_{N}, \bar{\alpha}_{N}\right) \leq 1, \text { a.s. }
$$

Now let

$$
\theta_{N}\left(a_{N}, R_{N}\right)=\sup _{0 \leq s \leq 1-a_{N}}\left\{R_{N}\left(s+a_{N}\right)-R_{N}(s)\right\} .
$$

By Lemma 2, we have for large $N$ that

$b_{N}^{-1} \sup _{0 \leq s \leq 1-a_{N}}\left|A_{N 2}\left(s+a_{N}\right)-A_{N 2}(s)\right| \leq N^{\frac{1}{2}}\left|1-\mu_{n}\right| b\left(a_{N}\right)(1+o(1))$, a.s.

Thus if (Q3) and $(Q 4)$ are satisfied, we get

$$
\theta_{N}\left(a_{N}, \bar{\alpha}_{N}\right) \leq \theta_{N}\left(a_{N}, A_{N 1}\right)+o(1) \text {, a.s., as } N \rightarrow+\infty .
$$

Furthermore it may be derived from Theorem 0.2 of Stute [10 that $(S 1),(S 2)$ and $(S 3)$ yield

$b_{N}^{-1} \sup _{0 \leq s \leq 1-a_{N}}\left|\gamma_{N}\left(\psi(s)+o\left(a_{N}\right)\right)-\gamma_{N}\left(\psi(s)+a_{N}\right)\right|=o(1)$, a.s., as $N \rightarrow+\infty$

It follows from (3.7) and (3.8) that $(S 1-2-3)$ and $(Q 1-3-4)$ together imply

$b_{N}^{-1} \theta_{N}\left(a_{N}, \bar{\alpha}_{N}\right) \geq b_{N}^{-1}\left\{\sup _{0 \leq s \leq 1-a_{N}} \gamma_{N}\left(\psi(s)+a_{N}\right)-\gamma_{N}(\psi(s))\right\}+o(1)$ a.s,

as $N \rightarrow+\infty$. Since $\psi:\left(0,1-a_{N}\right) \rightarrow\left(0, \psi\left(1-a_{N}\right)\right)$, is a bijection and since $\psi\left(1-a_{N}\right)=1-a_{N}(1+o(1))$,a.s., we may use Lemma 1 (formulas (2.8) and (2.9) when $(Q 1)$ holds to find for any $\varepsilon>0$, for any elementary event $\omega$, an $N_{o}(\omega)$ such that

$$
N>N_{o} \Rightarrow b_{N}^{-1} \theta_{N}\left(a_{N}, \bar{\alpha}_{N}\right) \geq \sup _{0 \leq s \leq 1-a_{N}(1+\varepsilon)}\left(\gamma_{N}\left(s+a_{N}\right)-\gamma_{N}(s)\right)+o(1) \text {, a.s. }
$$

Once again, we use the Theorem 02 of [10] to see that, under (S1-2-3), we have 


$$
\lim _{N \rightarrow+\infty} \sup _{0 \leq s \leq 1-a_{N}(1+\varepsilon)}\left\{\frac{\left|\gamma_{N}\left(s+a_{N}+\varepsilon a_{N}\right)-\gamma_{N}\left(s+a_{N}\right)\right|}{b_{N}}\right\} \leq \varepsilon^{\frac{1}{2}} \text {, a.s. }
$$

Thus, under (Q1-3-4) and for large values of $\mathrm{N}$, we get

$$
b_{N}^{-1} \theta_{N}\left(a_{N}, \bar{\alpha}_{N}\right) \geq b_{N}^{-1} \theta_{N}\left(a_{N}, \gamma_{N}\right)-\left(1+\frac{1}{2}\right) \varepsilon^{\frac{1}{2}}, a . s .,
$$

Hence Lemma 2.13 in [10] and (3.9) together yield

$$
\forall \varepsilon>0, \lim _{N \rightarrow+\infty} \inf \theta_{N}\left(a_{N}, \bar{\alpha}_{N}\right) \geq(1-\varepsilon)^{\frac{1}{2}}-\left(1+\frac{1}{2}\right), \text { a.s. }
$$

Finally (3.5) and (3.10) together ensure that

$$
\lim _{N \rightarrow+\infty} k_{N}\left(a_{N}, \bar{\alpha}_{N}\right)=1, \text { a.s. }
$$

whenever $(Q 1-3-4)$ and $(S 1-2-3)$ hold. But since $\log n \sim \log N$ ( $\mathrm{k}$ being fixed), one has

$$
\begin{gathered}
\left(\frac{\log \log n}{n}\right)^{\frac{1}{2}} \log a_{N}^{-1} \sim k^{\frac{1}{2}}\left(\frac{\log \log N}{\log a_{N}^{-1}}\right)^{\frac{1}{2}} \frac{\left(a_{N}^{\frac{1}{2}} \log a_{N}^{-1}\right)^{\frac{1}{2}}}{\left(N^{\frac{1}{2}} a_{N}^{\frac{1}{2}}\right)} \\
\left(\frac{\log \log N}{N a_{N} \log a_{N}^{-1}}\right)^{2} \log a_{N}^{-1} \leq \frac{(\log \log N)^{2}}{\left(\log a_{N}^{-1}\right)^{2}} \\
(2 \log \log n)\left(a_{N} \log a_{N}^{-1}\right) \sim\left(\frac{\log \log N}{\log a_{N}^{-1}}\right)\left(a_{N}^{\frac{1}{2}} \log a_{N}^{-1}\right)^{\frac{3}{2}}
\end{gathered}
$$

for large N. (2.1), (2.2) and (3.13) show that (S1) and (S2) imply (Q1$2-3)$ and this completes the proof of part I of Theorem 1.

\section{Proof of Part II of Theorem 1.}

The proof is the same as that of the first part. We only notice that if $a_{N}=c N^{-1} \log N, c>0,(Q 1-3-4)$ are satisfied for a fixed $\mathrm{k}$. To get Part II of Theorem 1, we use Theorem 1 (Part I) of [7] for the inequality " $\leq$ " and the Erdös-Renyi law for the increments of the uniform empirical process due to Komlos et al., and [4] for the inequality " $\geq "$. Similarly to the first case, we get an analogue to (3.9). That is, for any $\varepsilon>0$, for any elementary event $\omega$, we can find an $N_{1}(\omega)$ such that 


$$
\begin{gathered}
N>N_{1} \Rightarrow k_{N}\left(a_{N}, \bar{\alpha}_{N}\right) \geq \sup _{0 \leq s \leq 1-a(1+\varepsilon)}\left|\frac{\gamma_{N}(s+a(1+\varepsilon))-\gamma_{N}(s)}{b_{N}}\right| \\
-2 \varepsilon^{\frac{1}{2}} h(c \varepsilon)+\varepsilon^{\frac{1}{2}}
\end{gathered}
$$

where for any $\mathrm{s}, h(s)=\left(\frac{s}{2}\right)^{\frac{1}{2}}\left(\beta^{+}(s)-1\right)$ and $\beta^{+}(s)$ is the unique solution of the equation $x(\log x-1)+1=s^{-1}$ such that $\beta^{+}(s) \geq 1$. Now, since for any $f(),. g(),. \mathrm{K}$,

$\sup _{s e K} \max (f(s), g(s))=\max \left(\sup _{s \in K} f(s), \sup _{s \in K} g(s)\right)$ and $|x|=\max (x,-x)$

and since (see e.g. the third formula that follows Statement 11 in [7])

$\forall \varepsilon>0, \lim _{N \rightarrow+\infty} \inf \frac{\left|\gamma_{N}(s+a(1+\varepsilon))-\gamma_{N}(s)\right|}{b_{N}}=(1+\varepsilon)^{\frac{1}{2}} h((1+\varepsilon) c)$, a.s.,

then (3.14) implies that

$\forall \varepsilon>0, \lim _{N \rightarrow+\infty} \inf k_{N}\left(a_{N}, \bar{\alpha}_{N}\right) \geq(1+\varepsilon)^{\frac{1}{2}} h((1+\varepsilon) c)-2^{\frac{1}{2}} h(c \varepsilon)-\varepsilon^{\frac{1}{2}}$, a.s.

Thus it suffices to prove that : (2.1) for each fixed c, $h((1+\varepsilon) c) \rightarrow$ $h(c)$ as $\varepsilon \rightarrow 0$, and : (2.2) for each fixed $c$, $\varepsilon^{\frac{1}{2}} h(c \varepsilon) \rightarrow 0$ as $\varepsilon \rightarrow 0$. But these two points may be directly obtained by simple considerations.

Proof of part III of Theorem 1 .

The proof is very similar to that of Part I of Theorem 1. If suffices to remark that part III of Theorem 1 in [7] holds in the general case where $a_{N}=\alpha(\log N)^{-c}, c>0, \alpha>0$.

\section{Proof of Part IV of Theorem 1 .}

Here $a_{N}=c_{N} N^{-1} \log N, c_{N} \rightarrow 0$ as $N \rightarrow+\infty$. Let $d_{N}=N^{\frac{1}{2}}(\log N)^{-1} \log c_{N}^{-1}$. On the one hand, we have

(A)

$\left(N^{-1} \log \log N\right)^{\frac{1}{2}} \log a_{N}^{-1} \sim\left(N^{-1} \log \log N\right)^{\frac{1}{2}} \log c_{N}^{-1}+N^{-\frac{1}{2}}(\log \log N)^{\frac{1}{2}} \log N$, 


$$
\begin{aligned}
& \frac{(\log \log n)^{\frac{1}{2}}}{c_{N} \log N}\left(\log a_{N}^{-1}\right)^{-1} \sim \frac{(\log \log N)^{\frac{1}{2}}}{\left(\log c_{N}^{-1}\right) c_{N} \log N+c N(\log N)^{2}(1+o(1))} \\
& \text { (B) } \quad=\frac{(\log \log N)^{\frac{1}{2}}}{\log N}\left(c_{N} \log N\right)^{-1}\left(1+\frac{\log c_{N}^{-1}}{\log N}+o(1)\right) \\
& \text { (C) }\left((\log \log n)^{\frac{1}{2}}\left(a_{N} \log a_{N}^{-1}\right)^{\frac{1}{2}}\right)^{2}
\end{aligned}
$$

$=c_{N}\left(\log c_{N}^{-1}\right) N^{-1}(\log N)(\log \log N)+c_{N} N^{-1}(\log N)^{2}(\log \log n)(1+o(1))$.

Obviously $(A),(B)$ and $(C)$ together imply that the conditions of Part IV of Theorem 1, namely, as $N \rightarrow+\infty$,

$$
\begin{gathered}
c_{N} \rightarrow 0, \\
c_{N} \log N \rightarrow+\infty
\end{gathered}
$$

and

$$
(\log N)^{-1}\left(\log c_{N}^{-1}\right)(\log \log N) \rightarrow 0
$$

In turn these facts imply the conditions (Q1-3-4). On the other hand, we have

$$
\begin{gathered}
d_{N} \wedge_{N}\left(a_{N}, \bar{\alpha}_{N}\right) \leq d_{N} \wedge_{N}\left(a_{N}, A_{N 1}\right) \\
+0\left(\frac{\left(a_{N} \log a_{N}^{-1}\right)(\log \log n)^{\frac{1}{2}} N^{\frac{1}{2}} \log c_{N}^{-1}}{k^{\frac{1}{2}} \log N}\right)+0\left(\frac{\log \log n}{\log N} \log c_{N}^{-1}\right),
\end{gathered}
$$

a.s., as $N \rightarrow+\infty$, where we have used Lemma 2 and (3.2). Further, as $N \rightarrow+\infty$,

$$
\frac{\left(a_{N} \log a_{N}^{-1}\right)(\log \log n)^{\frac{1}{2}} N^{\frac{1}{2}} \log c_{N}^{-1}}{k^{\frac{1}{2}} \log N} \sim \frac{\left(\log a_{N}^{-1}\right)(\log \log N)(\log N)^{3}}{(\log N)\left(k^{\frac{1}{2}} \log N\right) N} c_{N} \rightarrow 0
$$

by the definition of $a_{N}$ and by $(W 1)$ and (W2). Thus, as $N \rightarrow+\infty$, we have

$$
d_{N} \wedge_{N}\left(a_{N}, \bar{\alpha}_{N}\right) \leq d_{N} \wedge_{N}\left(a_{N}, A_{N 1}\right)+o(1) \text {, a.s. }
$$


At this step, we apply Part II of Theorem 1 of [7] by using Lemma 1 which is true on account of $(Q 1)$.

\section{Proof of Theorem 2}

We shall omit details of the proofs of the different parts that are the same as those of the parts of Theorem 1. The only problem concerns the bounds depending on $\mathrm{k}$. However, this problem is solved by Lemmas 3 and 4 . Hence we only provide the following remarks.

(R1) In our different choices of $\left(a_{N}\right)$, we have that $N a_{N} \rightarrow+\infty$, as $N \rightarrow+\infty$.

(R2) If $a_{N} \leq t_{k}(\delta), \delta>2$, then for any $y>0$, there exists $k_{y}$ such that

$$
\forall k>k_{y}, \quad k^{-y} N \leq\left(N t_{k}\right)^{-1} \leq\left(N a_{N}\right)^{-1} \rightarrow 0 \text { as } N \rightarrow+\infty .
$$

(R3) $(\log \log n)=(\log \log N)(1+o(1))$ and $\log n=(\log N)(1+o(1))$, as $N \rightarrow+\infty$.

With these remarks, it is easily seen, as in the proof of Theorem 1 that the conditions $(K),(Q 2),(Q 3),(Q 4)$ and $(Q 5)$ are satisfied at the same time with the specific assumptions of each part of Theorem 2 as follows.

(a) (Q2) and $(K)$ are always satisfied if $a_{N}=t_{k}$. Indeed,

$$
k N^{-1} \log \log n \sim\left(k N^{\frac{1}{2}}\right)\left(N^{-\frac{1}{2}} \log \log N\right) \rightarrow 0 \text { as } N \rightarrow+\infty,
$$

by $(R 2)$ and $(K)$ and

$k(\log \log n)\left(\log a_{N}^{-1}\right) \sim\left(N^{-\frac{1}{4}} k \log a_{N}^{-1}\right)\left(N^{-\frac{1}{4}} \log \log N\right)\left(N a_{N}\right)^{-\frac{1}{4}} \rightarrow$, by $(R 2)-(R 3)$ and $(Q 2$.

(b) In Parts I, II and III of Theorem 2, the implication $\{(S 1),(S 3)\} \Rightarrow$ $\{(Q 3),(Q 4)\}$ is true whenever $\log \log n \sim \log \log N$ (see the lines that follow Formula (3.10) $)$ and $\left(N a_{N}\right) \rightarrow+\infty$ as $N \rightarrow+\infty$, which are derived from $(R 1),(R 2)$ and $(R 3)$.

(c) In Part IV, (Q5) is true independently of the behavior of $k$. 
Thus we may use Lemmas 3 and 4 instead of Lemmas 1 and 2 in the proofs of Theorem 1 to get the results of Theorem 2 in the same way. 


\section{Proof of The Corollary.}

This is a direct consequence of Theorems 1 and 2 and of Lemma 5. For Part III, the methods used in Part I of Theorem 1 must be repeated.

Remark 4. By letting $(N \log \log N)^{\frac{1}{4}}(\log N)^{\frac{1}{2}}\left(2 a_{N} \log a_{N}^{-1}\right)^{-\frac{1}{2}} \rightarrow 0$, it would be possible to derive part I, III and IV of the Theorem 1 from invariance principles such as in [1] or [5]. But the necessary amount of work would be unchanged relatively to our method. 


\section{REFERENCES}

[1] Aly, E.E.A., Beirlant, J. and Horvàth, L.(1984). Strong and weak approximation of the k-spacings processes. Z. Wahrsch. verw. Gabiete, 66, 461-484.

[2] Csőrgö, M. and Révèsz, P. (1981). Strong Approximation in Probability and Statistics. Academic Press. New York.

[3] Deheuvels, P. (1984). Spacings and applications. Techn. report 13, LSTA, Université Paris 6.

[4] Kómlos, J. Major, M. and Tusnàdy, G. (1975). Weak convergence and embedding. In : Colloquia Math. Soc. Janos. Boylai. Limit theorems of probability Theory, 149-165. Amsterdam, North-Holland.

[5] Lo, G.S. (1986). A strong upper bound in an improved approximation of the empirical k-spacings process and strong limits for the oscillation modulus. Techn. report 47, L.S.T.A., Université Paris 6.

[6] Loève, M. (1963). Probability Theory. Van Nostrand Comp. Inc. Princeton., 3rd ed.

[7] Mason, D.M., Shorack, G. and Wellner, A. (1983). Strong limit theorems for oscillation muduli of the empirical process. Z. Wahrsch. verw. Gabiete, 65, 83-87.

[8] Pyke, R. (1972). Spacings. J. Roy. Statist. Soc. Ser. B, 27, 359-449.

[9] Shorack, G.R. (1972). Convergence of quantile and spacing process with the application. Ann. Math. Statist., 43, 1400-1411.

[10] Stute, W. (1982). The oscillation behavior of empirical process. Ann. Probab., 10, 86-107. 